\title{
Free surface steady flow towards a gravity well
}

\author{
by T. D. Streltsova \\ Departmeat of (ivil Fngincerian \\ Universily of Bitmingham \\ (ringland)
}

\section{Introduction}

The form of the free surface near a pumped well and the extent of the seepage face in the well are not represented by hydraulic flow theory, which is based on the DupuitBoussinesq approximation. Since the main assumption of the Dupuit-Boussinesq approximation lies in ignoring vertical flow components, which leads to a uniform distribution of heads across any vertical of the flow, no allowance can be made for the presence of the seepage face. Its implication with respect to the free surface a failure to give the correct shape. The potential at an arbitrary point of the flow domain being distributed uniformly is replaced by the corresponding elevation of the free surface. By this approximation it is assumed also that the side of the well, exposed to the aquifer, admits water through the entire surface and offers no resistance to the entrance of water. The actual surface profile is considerably distorted within a distance about twice the thickness of the aquifer from the centre of the well. For practical purposes, however, it is important to know the real position of the water table elevations, as well as the elevation in the well, while the water is being pumped. The shape of the free surface is required and utilized for dewatering operations, while the current water level in the well is used to predict the safe yield for a given allowable drawdown and to choose the appropriate pumping equipment. Therefore, there is significant practical interest in discovering the relationship between the free surface level and the averaged head across any verticat of the flow.

The relationship, based on the consideration of the simplified "leakage" hypothesis, which takes into account vertical flow components, has been applied to radial well flow $[1,2,3]$, verifying the acceptability of this hypothesis. A free surface shape determination for the flow in the immediate vicinity of a fully penetrating pumping well under steady state conditions, with the vertical components taken in to account, is presented below.

\section{Definitions $\left({ }^{*}\right)$}

Assume that a well of radius $r_{w}$, penetrating completely an unconfined aquifer, is pumped at a constant discharge, $Q$, until a steady state is reached. The aquifer of infinite extent and finite thickness, $m$, is represented in the general case by an anisotropic water-bearing stratum with horizontal and vertical permeability, $k_{r}$ and $k_{z}$ respectively, which are assumed constant. Figure 1 shows the conditions of flow into such a well while water is being pumped. The

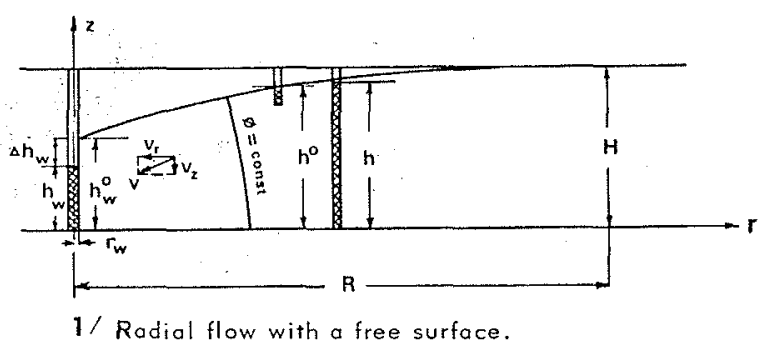

(*) The letter symbols are defined where they first appear. 
free surface is marked by the elevations, $h^{\circ}$, at which water will stand in boretioles positioned at or very near the free surface at distances $r$ from the centre of the pumped well. The water level in the well $h_{w}$ lies at some distance below the free surface $h_{w}^{\circ}$ outside the well, forming the

seepage face, $\Delta h_{w}$, as a vertical surface winere water seeps out of the soil into air. The boundary condition at the seepage face is given by assuming that the pressure is atmospheric (assumed zero).

In the axi-symmetric flow which is considered here the flow-nets, represented by the orthogonal nets of equipotential lines and streamlines, are deformable in the vicinity of the well at a distance of the order of $1.5 \mathrm{H}$ to $2 \mathrm{H}$, where $\mathrm{H}$ is the original depth of an undisturbed flow.

The piezometric head, $\phi=\rho, y+z$, which is the sum of the pressure head and the potential head, is dependent upon the height above datum. The equipotentials $\phi \ldots$ const are curvilinear. The averaged value of the piezometric head $\phi(r, z)$ atong any vertical is introduced as the value $h$,

$$
h=\frac{1}{h \circ} \int_{0}^{h^{\circ}} \phi(r, z) d z,
$$

which is the observed head in a well screened throughout and completely penetrating the unconfined aquifer.

The specific flux vector, $v$, i.e. flux per unit crosssection of flow, has two components: radial $v_{r}$ in the direction of $r$ decreasing, and axial or vertical $v_{z}$ in the direction of $z$ decreasing. Accordingly, the specific hydraulic resistance, $\phi$, which is inversely proportional to the flux of the liquid and is defined as a ratio of driving heads $\left(\phi_{1}-\phi_{2}\right)$ to the flux $v_{1,2}$ also has two components, radial, $\phi_{\mathrm{r}}$, and vertical, $\phi_{z}$.

The equation of continuity can be used with some approximation to compute the vertical velocity components, $v_{z}$ ' as well as the vertical specific hydraulic resistances, $\phi_{z}$.

\section{Vertical flow components}

The continuity equation for the flow with axial symmetry in the general case of an anisotropic water-bearing medium for steady state may be written as

$$
k_{r}\left(\frac{\partial^{2} \phi}{\partial r^{2}}+\frac{1}{r} \frac{\partial \phi}{\partial r}\right)+k_{z} \frac{\partial^{2} \phi}{\partial z^{2}}=0
$$

The value of the vertical velocity component, $v_{z}$, which is given by

$$
v_{z}=k \frac{\partial \phi}{z z}
$$

may be obtained by the integrating equation (2) with respect to $z$ and since $V_{z}=\partial \phi / \partial z=0$ when $z=0$ at the impervious layer, equation (2) gives

$$
v_{z}=-k_{r} \int_{0}^{z}\left(\frac{\partial^{2} \phi}{\partial r^{2}}+\frac{1}{r} \frac{\partial \phi}{\partial r}\right) d z
$$

Letting then $A(r, z)=-\int_{0}^{z}\left(\frac{\partial^{2} \phi}{\partial r^{2}}+\frac{1}{r} \frac{\partial \phi}{\partial r}\right) d z^{\prime}$, the expression (4) can be written as

$$
v_{z}=k_{r} A(r, z)
$$

The following integration of (5) and (3) leads to an expression for the piezometric head value $\phi$ as

$$
\phi(r, z)=\frac{k_{r}}{k_{z}} \int_{0}^{z} A(r, z) d z^{\prime}+\phi_{z}=0
$$

It is now assumed that the piezometric head $\phi(r, z)$, which is a function of the radius vector from the axis of symmetry $r$ and the vertical coordinate $z$, may be replaced along each vertical by the averaged value of head $h(r)$, defined by the expression (1). The averaged head, which has been integrated over $z$ from zero to $h^{\circ}$ is no longer dependent on the coordinate $z$, but only on the depth of flow. Thus the integral magnitude of the piezometric head defined by expression (1), being independent of the vertical coordinate while the diagram of actual piezometric head distribution $\phi(r, z)$ is curvilinear, allows the flow to be estimated in the terms of the averaged values.

The average value of the head at any vertical according to (1) and (6) is

$$
h=\frac{A(r)}{2 \mu^{2}} \frac{1}{h^{\circ}} \int_{0}^{h^{\circ}} z^{2} d z+h h_{z=0}=\frac{A(r)}{6 \mu^{2}} h^{02}+h_{z=0}
$$

where

$$
A(r)=-\left(\frac{\partial^{2} h}{\partial r^{2}}+\frac{l}{r} \frac{\partial h}{\partial r}\right), \quad \mu=\frac{k_{r}}{k_{z}}
$$

Let the specific hydraulic resistance in the vertical direction over the whole of the thickness of the aquifer be

$$
\phi_{z}=\frac{h^{0}-h}{v_{z}}
$$

The free-surface head from (6) substituting $z=h^{\circ}$ and taking into account ( 7 a) may be found to be

$$
h^{\circ}=\frac{A(t)}{2 \mu^{2}} h^{\circ 2}+h_{z=0}
$$

and the difference of the free-surface head and the averaged head (7) at the vertical is

$$
h^{\circ}-h=\frac{A(r)}{\mu^{2}} \frac{h^{02}}{3}
$$


Finally, since from (3) substituting $z=h^{\circ}$

$$
v_{z}=k_{r} h^{\circ} A(r)
$$

the equation (8) may be reduced further by insertion of (10) and (11) as

$$
\phi_{z=h^{\circ}}=\frac{h^{\circ}}{3 k_{z}}
$$

\section{Utilization and verification of the method for practical examples}

Taking into account the vertical tlow components in the general balance of the flow near a gravity well, both the form of the free surface and the heads along the impervious base of the aquifer can be determined.

The examples of the steady radial flow to a gravity well, which are treated here, have been solved elsewhere by means of the relaxation technique by Boulton [4] and Hall [5]. The principal purpose in choosing these particular cases is to compare and to assess the proposed method of the vertical flow components inclusion with the relaxation solution since the latter is still superior to any other in providing an occurate solution to the problem considered.

The case utilized first is Boulton's numerical solution with the characteristics of flow as follows: a fully penetrating pumped well of radiu's $r=2.0 \mathrm{~m}$ is situated in a homogeneous and isotropic unconfined aquifer of original depth $H=71.2 \mathrm{~m}$. At a distance $R=104 \mathrm{~m}$ from the well the water level $H=71.2 \mathrm{~m}$ is maintained constant. The steady state has been reached with the water in the well down to the elevation of the bottom of the aquifer, $h_{w}=0.0 \mathrm{~m}$.

This example was chosen not only because detailed calculated values of the free surface location as well as the base pressure head were available, but also because this particular case, when the well water is level with the bottom of the aquifer, represents a hypothetical limiting case, with a maximum extent of the seepage face, causing the errors observed to be maximum throughout the range of possible water level dispositions in the well.

The flow is represented as a simplified flow pattern with two lines of horizontal resistances, along upper and lower streamline boundaries of the pattern, to the nodal points of which are joined vertical resistances, calculated for one third of the saturated depth of aquifer according to (12).

The magnitude of these resistances are calculated by the use of formulae which for the cace of radial flaw aro

$$
\phi_{r ; n, n+1}=\frac{1}{2 \pi k H} \log \frac{r_{n+1}}{r_{n}}
$$

$$
\phi_{z ; n}=\frac{1}{3} H \frac{1}{\pi k\left(r_{n+1}^{\prime 2}-r_{n}^{\prime 2}\right)}
$$

where $r_{n}, r_{n+1}$ are the horizontal distances from the wellaxis to the centres of neighbouring $n$ and $(n+1)$ segments of the flow pattern and $r_{n}^{\prime}, r_{n+1}^{\prime}$ are the horizontal distances from the well axis to the boundaries of the segment (fig. 2).

Note that horizontal resistances being presented by two lines along upper and lower streamline boundaries, have their magnitudes doubled.

Calculation of the numerical values of the free surface and base pressure head for the flow presented by the simplified scheme of resistances shown in fig. 3 may be performed using either digital computer methods or resistance network analogue techniques.

Results of the solution for the first example considered, as compared with the relaxation procedure used by Boulton, are given in table 1 and fig. 4. The comparison of relaxation analyses with the calculations by the method proposed is quite satisfactory. The difference in the location of the free surface, the maximum discrepancy of which does not exceed $2.5 \%$, is explained by the somewhat different shape of the free surface from that usually expected in the approach to the discharge face of the well rather than by the accuracy and the assumptions of the method considered.

The classical analysis (see, for example, Muskat [6], page 289) assumes the free surface near the well to be a streamline. On this basis the intersection of the free surface and the surface of seepage at its upper terminal occurs tangentially to the discharge face of the well. The assump-

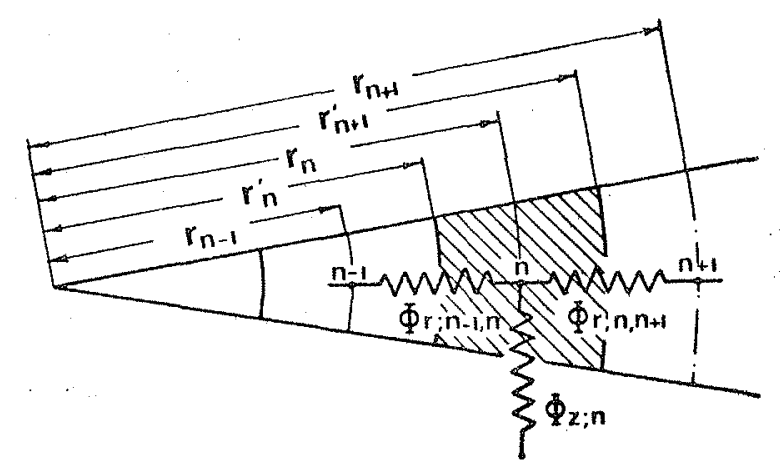

2/ Segment of radial flow with the hydraulic resistance components.

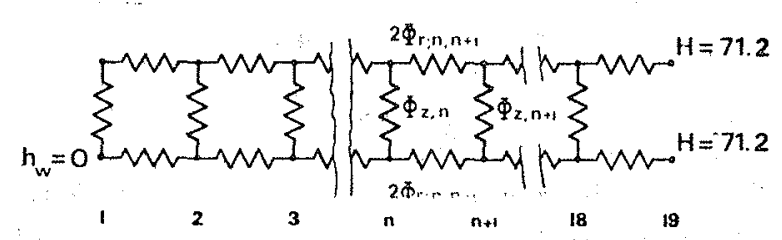

3 / The simplified flow scheme of resistances. 


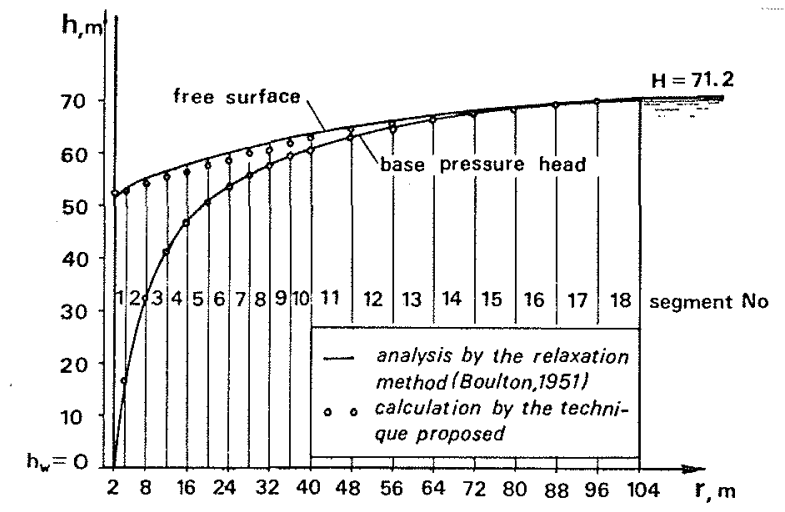

4 / Free surface and base pressure for the rodial flow under equilibrium conditions. (Boulton's example). tion, or rather simplification, of the free surface to be streamline eliminates any movements in a vertical direction. This proposition is more likely to be at variance with the conditions of actual cases. Such conditions arise for example when account is taken of replenishment due to any contribution from the region above the water table (leakage, yield from capillary fringe, rainfall etc.) or when applied to a submerged flow towards a partially screened well with a screen extending over the lower part of the aquifer, but it is these cases which are most likely to occur in practice.

The present analysis is based, as follows from the foregoing, on the assumption of the presence of the vertical flow components which, being a maximum at the well, decrease with increasing radius. The vertical velocities would make the curvature of the free surface convex downwards, tending towards the horizontal at the discharge face of the well.

An interesting investigation particularly in respect of the analysis of the free surface shape in the immediate vicinify of a gravity well has been carried out by Hall [5] using a series of large scale model tests as well as relaxation analysis of the corresponding cases. Attention has been paid in this investigation to the presence of the capillary effect. Yang's modification of the relaxation procedure in applying the method to the flow, when account is taken of the capillary layer, has been used in the analysis. On the basis of Hall's calculations the free surface tends towards a horizontal slope as it approaches the discharge face, meeting it at a right angle.

Fig. 5 presents the comparison of the results of Hall's calculations with the simplified resistances scheme solution for the case of radial flow having the following characteris. tics: a fully penetrating pumping well of radius $r_{w}=12.2 \mathrm{~cm}$ is situated in a homogeneous unconfined aquifer. At a radius $\mathrm{R}=195 \mathrm{~cm}$ from the well the water level $\mathrm{H}=121.92 \mathrm{~cm}$ is maintained constant. Equilibrium conditions have been reached with the water level in the well at $h_{w}=30.48 \mathrm{~cm}$. Hall's results included the effect of the capillary layer as a boundary condition, which gave a free surface profile with a reversed curvature (inset in fig. 5). Good agreement

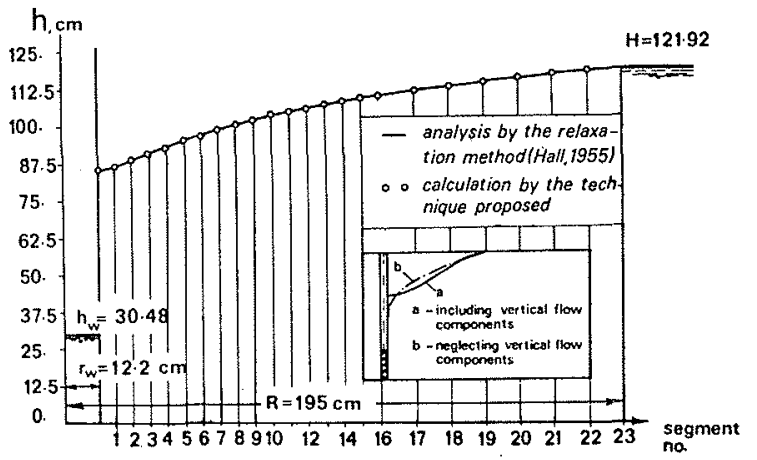

5/ Free surface for the radial flow under equilibrium conditions. (Hall's example).

is obtained between the two sets of results with the maximum percentage deviation not exceeding $1.5 \%$. Numerical values for the free surface position are given in table 2. In Hall's investigation the results were presented only as values of the head at nodal points of the relaxation network, therefore the values of the relaxation solution presented in table 2 are obtained by interpolation.

\section{References}

[1] SHESTAKOV (V.M.) and STRELTSOVA (T.D.). - Radial flow to a well completely penetrating an unconfined aquifer. Proceedings of the three seminars for the application of Geophysical and Mathematical methods in Hydrogeology and Engineering Geology, All-Union Research Institute of Hydrogeology and Engineering Geology, Moscow, (1970), p. 145-154 (in Russian).

[2] STRELTSOVA (T.D.). - Unsteady radial flow in an unconfined aquifer, Water Resources Research, Vol. 8, N4, August 1972.

[3] STRELTSOVA (T.D.). - The flow near a pumped well in an unconfined aquifer under non-steady conditions, Water Resources Research, 1973.

[4] BOULTON (N.S.). - The flow pattern near a gravity well in a uniform waterbearing medium, Journal of the Institution of Civil Engineers, London, Vol. 36, December 1951.

[5] HALL (H.P.)... An investigation of steady flow towards a gravity well, La Houille Blanche, N1, Jan-Feb. 1955, p. 8-35.

[6] MUSKAT (M). - The flow of homogeneous fluids through porous media, Mc Graw Hill Book Company, USA, (1946). 


\begin{tabular}{|c|c|c|c|c|c|c|c|}
\hline \multirow{2}{*}{$\begin{array}{c}\text { Segments } \\
\text { No., } \\
n\end{array}$} & \multirow{2}{*}{$\begin{array}{l}\text { Radius } r_{n} \\
\text { of the } \\
\text { segments }\end{array}$} & \multicolumn{3}{|c|}{ Free-surface values } & \multicolumn{3}{|c|}{ Base pressure values } \\
\hline & & $\begin{array}{c}\text { Relaxation } \\
\text { soln. }\end{array}$ & $\begin{array}{c}\text { Resistance } \\
\text { soln. }\end{array}$ & $\begin{array}{c}\% \\
\text { difference }\end{array}$ & $\begin{array}{c}\text { Relaxation } \\
\text { soln. }\end{array}$ & $\begin{array}{c}\text { Resistance } \\
\text { soln. }\end{array}$ & $\begin{array}{c}\% \\
\text { difference }\end{array}$ \\
\hline 1 & 2 & 51.53 & 52.52 & 1.39 & 0.00 & 0 & 0 \\
\hline 2 & 4 & 53.34 & 52.90 & 0.62 & 17.46 & 16.85 & 0.86 \\
\hline 3 & 8 & 55.42 & 54.01 & 1.98 & 32.89 & 32.75 & 0.20 \\
\hline 4 & 12 & 56.97 & 55.21 & 2.47 & 41.07 & 41.30 & 0.32 \\
\hline 5 & 16 & 58.23 & 56.48 & 2.46 & 46.41 & 46.87 & 0.65 \\
\hline 6 & 20 & 59.37 & 57.60 & 2.49 & 50.28 & 50.84 & 0.79 \\
\hline 7 & 24 & 60.44 & 59.55 & 1.25 & 53.27 & 53.62 & 0.49 \\
\hline 8 & 28 & 61.40 & 59.80 & 2.25 & 55.65 & 56.16 & 0.72 \\
\hline 9 & 32 & 62.30 & 60.80 & 2.11 & 57.60 & 58.08 & 0.57 \\
\hline 10 & 36 & 63.15 & 61.72 & 2.01 & 59.24 & 59.58 & 0.62 \\
\hline 11 & 40 & 63.91 & 62.59 & 1.85 & 60.64 & 61.04 & 0.56 \\
\hline 12 & 48 & 65.33 & 64.17 & 1.63 & 62.93 & 63.25 & 0.45 \\
\hline 13 & 56 & 66.48 & 65.54 & 1.32 & 64.74 & 64.98 & 0.34 \\
\hline 14 & 64 & 67.54 & 66.76 & 1.10 & 66.22 & 66.42 & 0.28 \\
\hline 15 & 72 & 68.41 & 67.84 & 0.80 & 67.48 & 67.63 & 0.21 \\
\hline .26 & 80 & 69.23 & 68.81 & 0.59 & 68.57 & 68.68 & 0.15 \\
\hline 17. & 88 & 69.94 & 69.68 & 0.37 & 69.53 & 69.61 & 0.11 \\
\hline 18 & 96 & 70.61 & 70.48 & 0.18 & 70.40 & 70.44 & 0.06 \\
\hline 19 & 104 & 71.20 & 71.20 & 0 & 71.20 & 71.20 & 0 \\
\hline
\end{tabular}

\begin{tabular}{|c|c|c|c|c|}
\hline \multirow{2}{*}{$\begin{array}{c}\text { Segments } \\
\text { No., } \\
n\end{array}$} & \multirow{2}{*}{$\begin{array}{c}\text { Radius } r_{n} \\
\text { of the } \\
\text { segments }\end{array}$} & \multicolumn{3}{|c|}{ Free-surface values } \\
\hline & & $\begin{array}{c}\text { Relaxation } \\
\text { soln.* }\end{array}$ & $\begin{array}{c}\text { Resistance } \\
\text { soln. }\end{array}$ & $\begin{array}{c}\% \\
\text { difference }\end{array}$ \\
\hline 1 & 12.2 & 86.40 & 86.27 & 0.10 \\
\hline 2 & 18.3 & 87.60 & 87.39 & 0.17 \\
\hline 3 & 24.4 & 90.00 & 89.09 & 0.74 \\
\hline 4 & 30.5 & 92.10 & 91.02 & 0.88 \\
\hline 5 & 36.6 & 94.18 & 93.02 & 0.95 \\
\hline 6 & 42.7 & 96.20 & 95.00 & 0.98 \\
\hline 7 & 48.8 & 98.50 & 96.90 & 1.31 \\
\hline 8 & 54.9 & 100.17 & 98.73 & 1.15 \\
\hline 9 & 61.0 & 102.26 & 100.46 & 1.47 \\
\hline 10 & 67.1 & 103.70 & 102.08 & 1.32 \\
\hline 11 & 73.2 & 105.20 & 103.62 & 1.29 \\
\hline 12 & $79 \cdot 3$ & 106.27 & 105.06 & 0.99 \\
\hline 13 & 85.4 & $107 \cdot 30$ & 106.42 & 0.72 \\
\hline 14 & 91.5 & 108.30 & 107.70 & 0.49 \\
\hline 15 & 97.6 & 109.50 & 108.90 & 0.49 \\
\hline 16 & 103.7 & 110.79 & 110.05 & 0.60 \\
\hline 17 & 109.8 & 111.78 & 111.13 & 0.53 \\
\hline 18 & 122.0 & 112.20 & 111.51 & 0.57 \\
\hline 19 & 134.2 & 114.20 & 113.47 & 0.52 \\
\hline 20 & 146.4 & 116.39 & 115.25 & 0.93 \\
\hline 21 & 158.6 & 117.72 & 116.86 & 0.70 \\
\hline 22 & 170.8 & 118.63 & 118.34 & 0.24 \\
\hline 23 & 183.0 & 120.25 & 119.70 & 0.45 \\
\hline $2 \%$ & 195.2 & 121.92 & 121.92 & 0.00 \\
\hline
\end{tabular}

Table 1

Comparison of results between the simplified resistances scheme solution and relaxation techniques (for the example of Fig. 4).

\section{Table 2}

Comparison of results between the simplified resistances scheme solution and relaxation techniques (for the example of Fig. 5).

* Note: The above relaxation solution values are obtained by interpolation from the diagram of Hall's Fig. 19. 